\title{
Long-tunnelled external ventricular drain as a long-term treatment option for hydrocephalus in a child with an unresectable low-grade supratentorial tumor: case report
}

\author{
John A. Emelifeonwu, MRCS, Drahoslav Sokol, MD, Pasquale Gallo, MD, \\ Jothy Kandasamy, FRCS, and Chandrasekaran Kaliaperumal, FRCSI, FRCSEd \\ Department of Paediatric Neurosurgery, Royal Hospital for Sick Children, Edinburgh, United Kingdom
}

The authors report a case of a child with hypothalamic-origin pilocytic astrocytoma and hydrocephalus, which was refractory to treatment with a ventriculoperitoneal shunt due to high CSF protein content. With parental education, the child's hydrocephalus was managed long-term in the community with a long-tunnelled external ventricular drain, which was maintained by his parents. To the authors' knowledge this is the first report of this management option as a longterm measure. No harm has come to the patient. The authors propose long-term, long-tunnelled external ventricular drain as a viable treatment option for such patients.

http://thejns.org/doi/abs/10.3171/2016.4.PEDS161

KEY WORDS long-tunnelled external ventricular drain; hydrocephalus; pilocytic astrocytoma; parental education

$\mathrm{T}$ UMORS arising from the CNS can block the normal CSF pathways, leading to hydrocephalus. This often necessitates a permanent CSF diversion procedure, for which insertion of a ventriculoperitoneal (VP) shunt is the most commonly performed. Some tumors, however, exude protein, leading to high CSF protein concentrations and an increased risk of shunt malfunction due to a combination of high CSF viscosity, peritoneal malabsorption, and occlusion of the shunt catheter by protein. ${ }^{8,10}$ The 1 -year malfunction rate for VP shunts is approximately $40 \%$, rising up to $50 \%$ at 2 years, ${ }^{4}$ and this is probably even higher in the context of persistently high CSF protein content. Children with unresectable low-grade tumors and shunted hydrocephalus with high protein content CSF may therefore be subjected to multiple shunt revisions over the course of their lifetime.

An alternative treatment strategy to VP shunts in these patients is the use of a long-term external ventricular drain (EVD). These devices also have high complication rates, with the overall complication rate ranging from $3.4 \%$ to $32.2 \%{ }^{7,15}$ The benefit of using EVDs is that they allow CSF flow to be interrogated without the need for an operation, and thus negate unnecessary general anesthetic, neurosur- gical intervention, and associated morbidities. Infection is the most common and worrisome complication; however, there is some evidence that the risk of EVD infections can be reduced if the externalized catheter is tunnelled some distance from the ventricle.

We describe the use of a long-tunnelled EVD (LTEVD) for long-term management of communicating hydrocephalus in a child with an unresectable hypothalamic low-grade glioma who had previously required multiple shunt revisions due to persistently highly proteinaceous CSF. With parental education the patient's hydrocephalus could be managed long term in the community with no infection.

\section{Case Report}

History and Examination

A 4-month-old baby boy was admitted to the Royal Hospital for Sick Children (RHSC) in Edinburgh for investigation of failure to thrive. On admission he was noted to have disconjugate eye movements, and a subsequent MRI scan of his head revealed a $5.2 \times 4.4 \times 5.2-\mathrm{cm}$ avidly enhancing suprasellar tumor (Fig. 1). A biopsy was obtained via

ABBREVIATIONS EVD = external ventricular drain; ICP = intracranial pressure; $L T E V D=$ long-tunnelled EVD; RHSC = Royal Hospital for Sick Children; VP = ventriculoperitoneal.

SUBMITTED January 13, 2016. ACCEPTED April 5, 2016.

INCLUDE WHEN CITING Published online June 3, 2016; DOI: 10.3171/2016.4.PEDS161. 

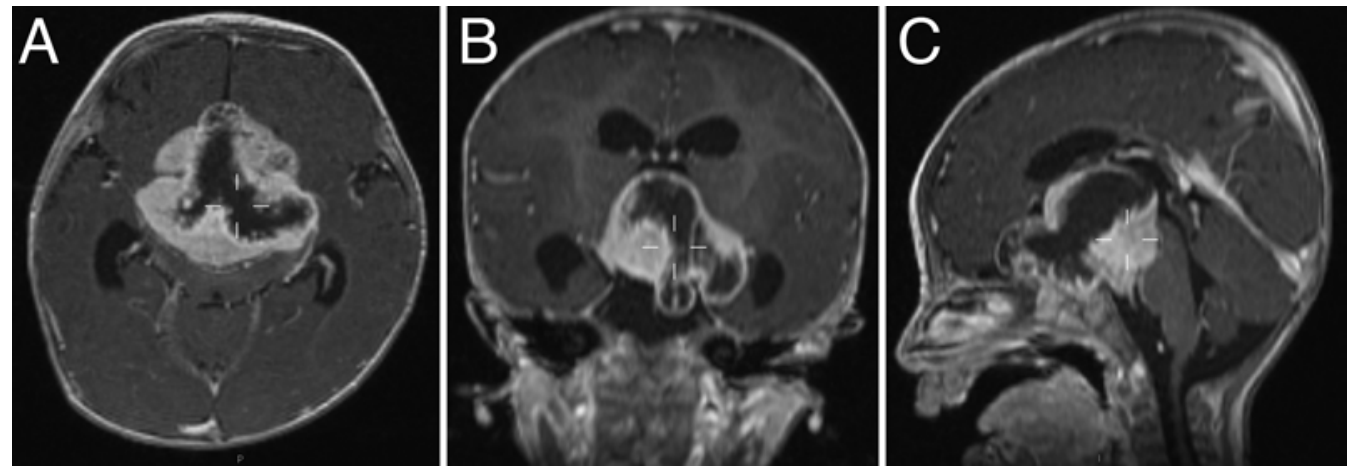

FIG. 1. Preoperative T1-weighted MRI scans (axial, coronal, and sagittal) obtained with gadolinium, showing the suprasellar lesion extending superiorly into the third ventricle and occluding both foramina of Munro. Prominent temporal ventricular horns suggest impending hydrocephalus.

an endoscopic right frontal transcortical, intraventricular approach, with a right frontal Ommaya reservoir left in situ in anticipation of future development of hydrocephalus. This led to the diagnosis of a hypothalamic-origin WHO Grade I pilocytic astrocytoma. A few days after this procedure, the child began to demonstrate evidence of raised intracranial pressure (ICP). This was confirmed by both a cranial ultrasound study, which revealed ventriculomegaly, and by direct measurement of ICP through the Ommaya reservoir. He therefore underwent insertion of a VP shunt, with the intracranial catheter inserted into the left occipital ventricle horn. One week after VP shunt insertion, he developed new evidence of raised ICP. A CT scan revealed that the shunt catheter had migrated out of the ventricle, necessitating a new VP shunt. He recovered well from this second operation.

\section{Initial Treatment}

The tumor was believed to be unresectable due to its size and location. The patient was treated with a 6-week course of chemotherapy (3 cycles of vincristine and carboplatin, with 2 concurrent cycles of temozolamide). Unfortunately, the tumor proved unresponsive to chemotherapy, and a decision was made by our pediatric neuro-oncology multidisciplinary team to commence palliative treatment, including CSF diversion to relieve raised ICP. Over the subsequent few months and years, he went on to have 12 VP shunt revisions over a 35-month period. His VP shunt was eventually converted to a ventriculoatrial shunt due to the frequent shunt blockages that were thought to be due to a persistently high CSF protein load. Two further revisions over 1 month were required after the first ventriculoatrial shunt was inserted; once as a consequence of shunt blockage, and on the second occasion, the intracranial catheter had migrated out of the ventricle. The reason for catheter migration is unknown but may have been due to changes in the compliance of the ventricles after so many cannulations.

Following discussions with the parents, a decision was made to insert an LTEVD as a long-term management strategy. The decision was again discussed, and was approved by the pediatric multidisciplinary team (consisting of the pediatric neurologist, oncologists, neurosurgeons, and microbiologist) at the RHSC. The proposed benefit of
LTEVD was that when a malfunction was suspected, catheter integrity and CSF flow could be interrogated externally, negating the risks and morbidities associated with surgical exploration and the exposure to additional radiation through imaging, procedures that are often required when investigating an internalized system. Also, because the child was wheelchair bound, it was believed that his limited self-mobility made him a suitable candidate for an LTEVD.

\section{Operation}

After induction of general anesthesia, a right frontal, antibiotic-impregnated, valveless EVD was inserted and tunnelled approximately $30 \mathrm{~cm}$ subcutaneously to the periumbilical region. Rigorous parental education on management of the EVD was provided in the days following its insertion, including how to change the EVD bag in a sterile manner, how to clamp the EVD at times of excess CSF flow, and how to recognize the red flag signs and symptoms for suspected infection. Following advice from the pediatric microbiologist, long-term prophylactic rifampicin was also prescribed. To date, there have been no side effects noted from the use of long-term rifampicin. The parents were given open access to the neurosurgical ward at the RHSC.

\section{Postoperative Course}

At the time of this writing, LTEVD had been used to manage the patient's hydrocephalus for a total of 250 days, with 1 revision due to catheter blockage (average duration of 125 days). There have been 4 admissions due to symptomatic catheter blockages. On these occasions, the presenting complaint has been drowsiness related to reduced or no CSF flow from the EVD catheter. On all occasions, the parents, who have a very good knowledge of the patient's symptomatology, were able to contact the on-call neurosurgery team in a timely manner, and CSF flow was reestablished by sterile flushing and aspiration to unblock the tubing. This has so far proved successful in reestablishing CSF flow. To date the patient has not developed ventriculitis. However, CSF was routinely sent for microbiological analysis as a means of surveillance, and on one occasion CSF cytology yielded a predominantly lymphocytic pleocytosis, with Staphylococcus epidermi- 
dis grown on subsequent cultures. Because the child was clinically well, we decided not to change his prophylactic antibiotic regimen.

\section{Discussion \\ Mechanism of Shunt Malfunction in CSF Containing High Protein}

Although well documented, the precise mechanism for the high protein content in the CSF of patients with gliomas is unknown. Reports of high protein-containing CSF in association with optic pathway/hypothalamic gliomas are particularly well documented in the literature. ${ }^{12,13,16}$ Various theories have been postulated, including the theory that tumors may directly exude proteins into the subarachnoid spaces. Elevated CSF protein is believed in turn to increase the incidence of VP shunt malformation, again through unclear mechanisms. Many theories have again been proposed, including the theory that protein clumps either disturb the valve mechanism or occlude the shunt lumen, and that high protein-containing CSF impairs peritoneal $a b-$ sorption of CSF. It should be noted that experimental models have failed to show that high protein-containing fluid compromises shunt performance. Indeed, some authors have suggested a beneficial effect of raised protein on shunt function, by inhibiting bacterial adhesion to the inside of the catheter. ${ }^{3}$ Our case, however, undoubtedly demonstrates poor shunt performance in the presence of raised CSF protein, with biochemical evidence of persistently raised CSF protein on each occasion when the patient's shunt was blocked. This propensity of VP shunts to malfunction in these patients suggests that internalized shunts are highly likely to fail, and to require multiple revisions.

\section{Reducing EVD Infections}

The alternative to VP shunts in patients with high protein-containing CSF is long-term EVDs. However, EVDs have high infection rates, which range from $3 \%$ to $32 \%$. These high rates of infection are multifactorial; thus, developing strategies to effectively reduce the rates of infections has proved difficult. There is, however, good evidence that certain steps can be taken to reduce the rate of infection, including the use of LTEVDs. Several authors have reported significant reductions in rates of infection with tunnelling, ,2,5 and both Collins et al. and Khanna et al. reported EVD durations of 40 days and 42 days, respectively, when significant tunnelling techniques are used. It is unknown why long subcutaneous tunnelling reduces infection. It may be due to the increased distances that bacteria have to migrate, or it may be that tunnelling inferiorly provides a path of lower resistance for CSF diversion and reduces the risk of a CSF leak through the craniotomy wound site, which may be a risk factor for EVD infection. ${ }^{6}$

In our reported case, the catheter of the EVD was tunnelled to the periumbilical area (approximately $30 \mathrm{~cm}$ ), with an average duration of 125 days (range 83-167 days) for the device. To our knowledge, these are the longest reported durations for inserted EVDs in the literature. If EVD infections are reduced by long tunnelling, then it should be considered an alternative treatment option in the management of hydrocephalus in children who are at high risk of shunt malfunction. External ventricular drains have a further advantage over internalized systems, in that they allow the CSF diversion catheter to be interrogated without the need for surgical exploration under general anesthesia. As we have stated, in our patient the blockages of the EVD catheter have been successfully managed on several occasions by sterile aspiration of CSF to unblock the catheter tubing. This also avoids the radiation associated with radiological imaging such as shunt series, which is a mainstay in investigating suspected blocked shunts, and the morbidity that would be associated with surgical exploration of an internalized system.

\section{Challenges of Managing LTEVDs in the Community}

Parental education has been successfully used to reduce patients' reliance on hospital services in the past. Examples include self-catheterization in patients with spina bifida and self-care of vascular access devices. However, with CSF diversion devices, worries about ventriculitis and subsequent significant morbidity have meant that internalized diversion (shunt placement) is the preferred treatment modality for palliating chronic hydrocephalus. With their high rate of malfunction, VP shunts can have significant impacts on quality of life, with frequent and prolonged hospitalization, and also with the risks involved in general anesthesia and surgical exploration to investigate or treat shunt malfunctions.

The cost to the health services is also significant. Health economy studies from the US have estimated the management of hydrocephalus in children to cost in excess of $\$ 100$ million of the national health care expenditure annually.,11 Children with hydrocephalus have been found to use a disproportionate share of hospital days and health care expenditure, exceeding other chronic diseases such as cystic fibrosis. ${ }^{14}$ In the case presented, the LTEVD reduced the rate of surgical exploration and hence reduced the associated morbidity to the child, as well as the financial burdens associated with in-patient investigations of suspected shunt malfunctions. Studies in other fields have shown that highly motivated parents are able to manage simple medical procedures effectively when provided with adequate medical support, thus reducing in-patient admission. We therefore propose LTEVDs with such support as a cost-effective treatment option in palliative management in children with unresectable low-grade gliomas and hydrocephalus that is refractory to treatment with internalized shunts.

\section{Conclusions}

Internalized VP shunts in the clinical scenario presented here have a high rate of malfunction due to infection and blockages, the latter being particularly problematic in optic pathway/hypothalamic gliomas. Long-term LTEVDs are an alternative, which in the past have been avoided because of worries about infection. We have shown that with parental motivation and education, and with appropriate medical support, long-term management of hydrocephalus with an LTEVD and appropriate prophylactic antibiotics is possible, and may limit the radiation, morbidities, and hospital stay associated with investigating and managing suspected shunt malfunction in these children. 


\section{References}

1. Bondurant CP, Jimenez DF: Epidemiology of cerebrospinal fluid shunting. Pediatr Neurosurg 23:254-259, 1995

2. Collins CDE, Hartley JC, Chakraborty A, Thompson DNP: Long subcutaneous tunnelling reduces infection rates in paediatric external ventricular drains. Childs Nerv Syst 30:1671-1678, 2014

3. Donlan RM, Costerton JW: Biofilms: survival mechanisms of clinically relevant microorganisms. Clin Microbiol Rev 15:167-193, 2002

4. Drake JM, Kestle JR, Tuli S: CSF shunts 50 years on-past, present and future. Childs Nerv Syst 16:800-804, 2000

5. Khanna RK, Rosenblum ML, Rock JP, Malik GM: Prolonged external ventricular drainage with percutaneous long-tunnel ventriculostomies. J Neurosurg 83:791-794, 1995

6. Korinek AM, Reina M, Boch AL, Rivera AO, De Bels D, Puybasset L: Prevention of external ventricular drain-related ventriculitis. Acta Neurochir (Wien) 147:39-46, 2005

7. Lozier AP, Sciacca RR, Romagnoli MF, Connolly ES Jr: Ventriculostomy-related infections: a critical review of the literature. Neurosurgery 51:170-182, 2002

8. Occhipinti E, Carapella CM: Shunt failure in hydrocephalus with high protein fluid. Front Neurol Neurosci 8:220-222, 1982

9. Omar MA, Mohd Haspani MS: The risk factors of external ventricular drainage-related infection at Hospital Kuala Lumpur: an observational study. Malays J Med Sci 17:4854,2010

10. Salmon JH: A ventriculo-peritoneal shunt for hemorrhagic or high protein fluid. Surg Neurol 8:69-70, 1977

11. Sciubba DM, Lin LM, Woodworth GF, McGirt MJ, Carson B, Jallo GI: Factors contributing to the medical costs of cerebrospinal fluid shunt infection treatment in pediatric patients with standard shunt components compared with those in patients with antibiotic impregnated components. Neurosurg Focus 22(4):E9, 2007

12. Shooman D, Vajramani GV, Davidson J, Sparrow OC: Use of intrathecal urokinase in repeated shunt and external ventric- ular drain blockage from high CSF protein due to an optic pathway glioma. Childs Nerv Syst 26:607-611, 2010

13. Shuper A, Horev G, Michovitz S, Korenreich L, Zaizov R, Cohen IJ: Optic chiasm glioma, electrolyte abnormalities, nonobstructive hydrocephalus and ascites. Med Pediatr Oncol 29:33-35, 1997

14. Simon TD, Riva-Cambrin J, Srivastava R, Bratton SL, Dean JM, Kestle JR: Hospital care for children with hydrocephalus in the United States: utilization, charges, comorbidities, and deaths. J Neurosurg Pediatr 1:131-137, 2008

15. Stenager E, Gerner-Smidt P, Kock-Jensen C: Ventriculostomy-related infections - an epidemiological study. Acta Neurochir (Wien) 83:20-23, 1986

16. West GA, Berger MS, Geyer JR: Childhood optic pathway tumors associated with ascites following ventriculoperitoneal shunt placement. Pediatr Neurosurg 21:254-259, 1994

\section{Disclosures}

The authors report no conflict of interest concerning the materials or methods used in this study or the findings specified in this paper.

\section{Author Contributions}

Conception and design: Emelifeonwu. Acquisition of data: Emelifeonwu. Analysis and interpretation of data: Emelifeonwu. Drafting the article: Emelifeonwu. Critically revising the article: Emelifeonwu, Kaliaperumal. Reviewed submitted version of manuscript: Emelifeonwu. Administrative/technical/material support: Kaliaperumal. Study supervision: Sokol, Gallo, Kandasamy, Kaliaperumal.

\section{Correspondence}

John A. Emelifeonwu, Department of Neurosurgery, Royal Hospital for Sick Children, 9 Sciennes Rd., Edinburgh EH9 1LF, United Kingdom. email: johnemelifeonwu@gmail.com. 